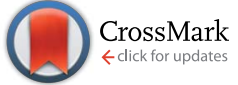

Cite this: RSC Adv., 2014, 4, 61086

Received 5th September 2014 Accepted 6th November 2014

DOI: $10.1039 / c 4 r a 09872 b$

www.rsc.org/advances

\section{The effect of hydroxytyrosol and its nitroderivatives on catechol-O-methyl transferase activity in rat striatal tissue}

\author{
Elena Gallardo, ${ }^{\text {ab }}$ Andrés Madrona, ${ }^{a}$ Rocío Palma-Valdés, ${ }^{a}$ Mariana Trujillo, ${ }^{a}$ \\ José Luis Espartero ${ }^{a}$ and Marti Santiago*b
}

\begin{abstract}
Hydroxytyrosol is a well-known phenolic compound with antioxidant properties that is found in virgin olive oil. Studies have shown that virgin olive oil has neuroprotective effects in rats; thus the purpose of the present study was to investigate the neuroprotective effect of hydroxytyrosol in rats. Additionally, this study aimed to investigate the neuroprotective potential of a homologous series of compounds with better lipophilic profiles in order to increase the assortment of compounds with a putative effect against Parkinson's disease (PD). In this context, the inhibition of catechol-O-methyl transferase (COMT) activity by hydroxytyrosol, nitrohydroxytyrosol, nitrohydroxytyrosol acetate and ethyl nitrohydroxytyrosol ether was investigated by measuring intracellular dopamine and its metabolite levels in the corpus striatum by high performance liquid chromatography (HPLC) with electrochemical detection. The animals received an acute (single dose; $20 \mathrm{mg} \mathrm{kg}^{-1}$; i.p.) or chronic (one daily dose for 5 days; $20 \mathrm{mg} \mathrm{kg}^{-1}$; i.p.) treatment of hydroxytyrosol and its nitroderivatives. For comparison, a commercial COMT inhibitor, Ro 41-0960, was also included. Our data show that acute and chronic systemic administration of these compounds produced a clear and statistically significant increase in the intracellular levels of dopamine and its metabolite, 3,4-dihydroxyphenylacetic acid. The increase in dopamine levels was very similar to the increase seen with Ro 41-0960 treatment. The effect of chronic treatment was stronger than that of acute treatment. With respect to the intracellular level of homovanillic acid, Ro 41-0960 produced a statistically significant decrease which it was not observed when hydroxytyrosol and its nitroderivatives were systemically administered. However, the chronic homovanillic acid treatment effect was stronger than the acute treatment. The results suggest that these compounds could inhibit COMT activity.
\end{abstract}

\section{Introduction}

Natural polyphenols present in the Mediterranean diet exert a variety of biochemical and pharmacological roles in several diseases; this has been especially documented in neurodegenerative disorders. Among virgin olive oil (VOO) phenols, hydroxytyrosol (HTy) stands out as a powerful antioxidant in both in vitro and in vivo studies, ${ }^{1}$ and a wide range of associated biological activities have been described for it. ${ }^{2}$ For example, HTy is a potent inhibitor of the low density lipoprotein (LDL) oxidation. ${ }^{2,3}$ HTy has the capacity to scavenge free radical species $^{4}$ as well as prompt the activity of antioxidant enzymes. ${ }^{5}$ These activities are central to cellular processes which protect against oxidative stress. Oxidative stress has been identified as one of the underlying factors of neurodegenerative diseases such as Parkinson's (PD). Furthermore, HTy modulates

aDepartment of Organic and Pharmaceutical Chemistry, Faculty of Pharmacy, University of Seville, 41012-Seville, Spain

${ }^{b}$ Department of Biochemistry and Molecular Biology, Faculty of Pharmacy, University of Seville, 41012-Seville, Spain. E-mail: msantiago@us.es neuroinflammation, ${ }^{6,7}$ another factor in PD progression and this effect is related to its aforementioned antioxidant properties.

Due to these remarkable biological properties, many HTy derivatives have been prepared in the past few years, and their structure/antioxidant activity relationships (SAR) have been studied. These studies have identified a key role of the free catechol in their function. ${ }^{8}$ Furthermore, acylation or alkylation of HTy with variable side chain lengths gives rise to lipophilic HTy esters ${ }^{9}$ and ethers, ${ }^{10}$ respectively. In the literature, most of the studies on lipophilic antioxidants reported an increase in the antioxidant activity for these derivatives as compared with free precursors. ${ }^{8-10}$ Moreover, some recent contributions revealed a nonlinear trend in this increase in emulsified, liposomal and cellular systems, with a maximum antioxidant activity for medium chain length, which lead to the cut-off effect theory. ${ }^{\mathbf{1 1 - 1 3}}$

Some reviews have been recently published concerning different biological activities of nitrocatecholic compounds, ${ }^{\mathbf{1 4 , 1 5}}$ as enzymatic inhibitors or antioxidants. ${ }^{\mathbf{1 6}}$ Among these nitrocatechols are tolcapone ${ }^{17}$ and entacapone, ${ }^{18}$ which are highly effective as inhibitors of catechol-O-methyl transferase (COMT), which are part of the second-generation of COMT inhibitors 

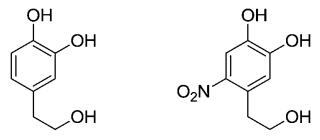

$\mathrm{NO}_{2} \mathrm{HTy}$

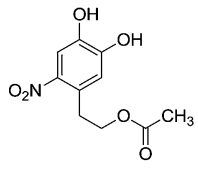

$\mathrm{NO}_{2} \mathrm{HTy}-\mathrm{A}$

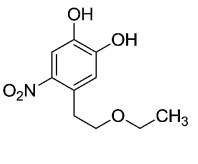

$\mathrm{NO}_{2} \mathrm{HTy}-\mathrm{E}$
Fig. 1 Chemical structure of hydroxytyrosol (HTy), nitrohydroxytyrosol $\left(\mathrm{NO}_{2} \mathrm{HTy}\right)$, nitrohydroxytyrosyl acetate $\left(\mathrm{NO}_{2} \mathrm{HTy}-\mathrm{A}\right)$ and ethyl nitrohydroxytyrosyl ether $\left(\mathrm{NO}_{2} \mathrm{HTy}-\mathrm{E}\right)$.

used in the treatment of PD. Although they were approved and reached the marketplace as adjuncts to levodopa therapy, tolcapone was subsequently withdrawn due to hepatotoxicity concerns ${ }^{18-20}$ Entacapone unfortunately has a very short in vivo half-life and, thus, its clinical efficacy has been questioned. ${ }^{21}$ Thus, given the lack of pharmacological availability of COMT inhibitors, there has been a great effort in recent years in drug discovery and the development of new selective and potent COMT inhibitors with safer and long-acting profiles. ${ }^{22-25}$

For these reasons, nitroderivatives of HTy, nitrohydroxytyrosol $\left(\mathrm{NO}_{2} \mathrm{HTy}\right)$, nitrohydroxytyrosyl acetate $\left(\mathrm{NO}_{2} \mathrm{HTy}-\mathrm{A}\right)$ and ethyl nitrohydroxytyrosyl ether ( $\mathrm{NO}_{2} \mathrm{HTy}$-E) (Fig. 1), which contain a catechol pharmacophore with a highly electronegative nitro group at ortho-position relative to the side-chain substituent, have been synthesized as a new class of potential COMT inhibitors. These inhibitors could play a role in the prevention of PD. Thus, recent work has examined the syntheses of some nitroester derivatives of HTy with variable acyl side chain lengths from 2 to 16 carbon atoms and has also evaluated their antioxidant activity by FRAP, ABTS, and ORAC assays. ${ }^{26}$ These nitrocatechols, obtained from HTy recovered from olive oil wastewaters, have demonstrated a remarkable antioxidant capacity as reducing agents and free radical scavengers ${ }^{26}$ Moreover, they did not show any cytotoxic effect in human HepG2 cells, thus they have the value of protecting against oxidative stress without any toxicity concerns. $^{27}$

Considering the above evidence, nitroderivatives of HTy have been proposed as potential and suitable options for the prevention of PD. Therefore, the aim of the present study was to evaluate the effect of HTy and its nitroderivatives on COMT activity in the rat's brain, by measuring the striatal content of intracellular dopamine (DA) and its metabolites, 3,4-dihydroxyphenylacetic acid (DOPAC) and homovanillic acid (HVA), using HPLC with electrochemical detection. A known commercial inhibitor, Ro 41-0960 was also included in our study, in order to compare data with a typical COMT inhibitor.

\section{Results and discussion}

\section{Dopamine (DA)}

The acute treatment with a single systemic administration $\left(20 \mathrm{mg} \mathrm{kg}^{-1}\right.$, i.p.) showed a clear and significant increase in the striatal content of DA when Ro 41-0960 (123.6\%), HTy (118.9\%) and $\mathrm{NO}_{2} \mathrm{HTy}(119.6 \%)$ were compared to saline (Fig. 2). However, $\mathrm{NO}_{2} \mathrm{HTy}-\mathrm{A}(105.8 \%)$ and $\mathrm{NO}_{2} \mathrm{HTy}-\mathrm{E}(104.8 \%)$ showed only small increases, which were not statistically significant

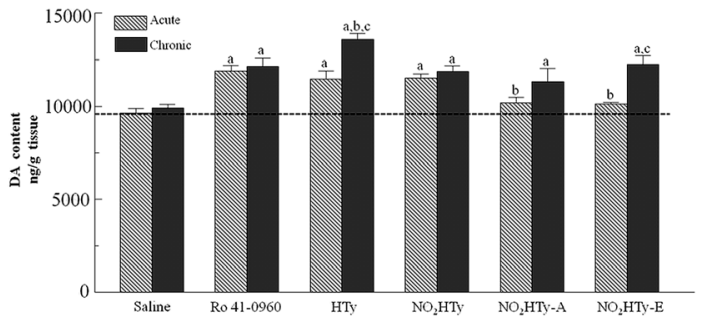

Fig. 2 Effect of the acute and chronic systemic treatments on the striatal content of DA. Each column represents mean \pm SEM (vertical lines), expressed as $n g ~^{-1}$ wet tissue $(N=4-8)$. Statistical significance: (a) compared with saline treatment, (b) compared with Ro 41-0960 treatment, and (c) compared with the same treatment under acute conditions. See text for statistical details.

with respect to saline, but statistically significant when they were compared to Ro 41-0960 (Fig. 2).

Chronic systemic administration produced an increase in the striatal content of DA, which was higher and statistically significant than that produced by the acute systemic administration in $\mathrm{HTy}(137.0 \%)$ and $\mathrm{NO}_{2} \mathrm{HTy}-\mathrm{E}$ (123.6\%) (Fig. 2). However, $\mathrm{NO}_{2} \mathrm{HTy}(113.7 \%)$ and $\mathrm{NO}_{2} \mathrm{HTy}-\mathrm{A}(114.3 \%)$ chronic treatment did not show any statistical difference when compared with acute treatment (Fig. 1). Ro 41-0960 chronic systemic administration produced similar increase (122.5\%) when compared with the acute systemic administration (Fig. 2).

\section{3,4-Dihydroxyphenylacetic acid (DOPAC)}

Both acute and chronic systemic administrations produced a strong and significant increase in the striatal content of DOPAC (Fig. 3). The biggest increase in the striatal content of DOPAC was found when Ro 41-0960 was systemically administered (acute, 234.6\% and chronic, 225.5\%; Fig. 3). HTy systemic administration produced very similar increases in the striatal content of DOPAC for both treatments (acute, 152.7\% and chronic, $155.1 \%$ ). $\mathrm{NO}_{2} \mathrm{HTY}$-A and $\mathrm{NO}_{2} \mathrm{HTy}$-E systemic administrations produced a bigger and statistically significant increase in the striatal content of DOPAC when chronic treatment was compared with acute treatment: $\mathrm{NO}_{2} \mathrm{HTy}-\mathrm{A}$ (acute, $146.7 \%$ and chronic, $181.8 \%$ ) and $\mathrm{NO}_{2} \mathrm{HTy}-\mathrm{E}$ (acute, $122.7 \%$

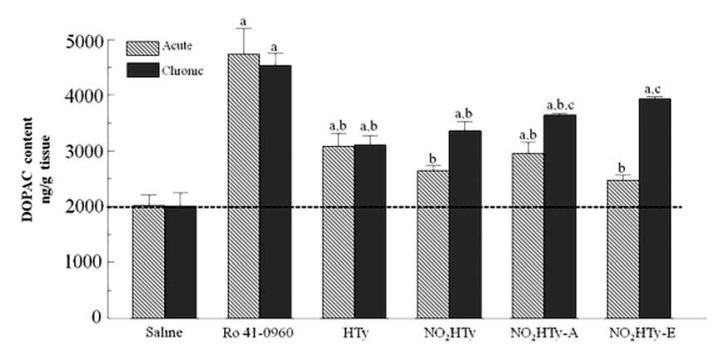

Fig. 3 Effect of the acute and chronic systemic treatments on the striatal content of DOPAC. Each column represents mean \pm SEM (vertical lines), expressed as $\mathrm{ng} \mathrm{g}^{-1}$ wet tissue $(N=4-8)$. Statistical significance: (a) compared with saline treatment, (b) compared with Ro 41-0960 treatment, and (c) compared with the same treatment under acute conditions. See text for statistical details. 
and chronic, 195.8\%) (Fig. 3). However, $\mathrm{NO}_{2} \mathrm{HTy}$ systemic administration produced a high increase in the striatal content of DOPAC, which was not statistically significant (acute, 131.3\% and chronic, 167.6\%) (Fig. 3).

$\mathrm{HTy}, \mathrm{NO}_{2} \mathrm{HTy}, \mathrm{NO}_{2} \mathrm{HTy}-\mathrm{A}$ and $\mathrm{NO}_{2} \mathrm{HTy}-\mathrm{E}$ acute and chronic systemic administration were statistically significant when compared with Ro 41-0960, with the exception of the $\mathrm{NO}_{2} \mathrm{HTy}-\mathrm{E}$ chronic systemic administration (Fig. 3).

\section{Homovanillic acid (HVA)}

Lastly, striatal content of HVA differed significantly when comparing Ro 41-0960 with phenolic compounds. In fact, Ro 410960 systemic administration showed a strong reduction in the striatal content of HVA, while no decrease was found with the systemic administration of the other compounds (Fig. 4). However, in all compounds studied, a clear reduction in the striatal content of HVA was found when comparing chronic and acute systemic administration. In these experiments, the biggest difference was found with systemic Ro 41-0960 treatment: acute, $58.0 \%$ and chronic, $\mathbf{1 3 . 1 \%}$ (Fig. 4). Similar results, but to a lesser extent, were found with HTy (acute, $138.3 \%$ and chronic, $102.5 \%$ ); $\mathrm{NO}_{2} \mathrm{HTy}$ (acute $114.9 \%$ and chronic, $95.7 \%$ ); $\mathrm{NO}_{2} \mathrm{HTy}-\mathrm{A}$ (acute, $107.7 \%$ and chronic, $83.9 \%$ ); and $\mathrm{NO}_{2} \mathrm{HTy}-\mathrm{E}$ (acute, $117.6 \%$ and chronic, $86.5 \%$ ) (Fig. 4).

The naturally occurring biophenol, HTy, along with some of its nitroderivatives, have been studied in order to demonstrate their potential neuroprotection against PD. This work is valuable in the light of emerging concerns about availability and relatively short half-lives of the current clinical COMT inhibitors $^{28,29}$ This work looked to identify compounds that could be used as potent and long-acting COMT inhibitors as an adjunct to L-DOPA/AADC inhibitor therapy for PD. ${ }^{30,31}$ Therefore, we have designed novel lipophilic hydroxytyrosyl derivatives as COMT inhibitors and we have evaluated their capability to cross the blood brain barrier and, their effects on the neurochemical markers of PD in rat striatal tissue. Considering the controversial nature of the proposed selectivity of COMT inhibitors $;^{30}$ we studied the potency of novel inhibitors of COMT activity in brain tissue after intraperitoneal administration.

In order to be considered a COMT inhibitor a compound must increase the amount of DA present in the brain due to

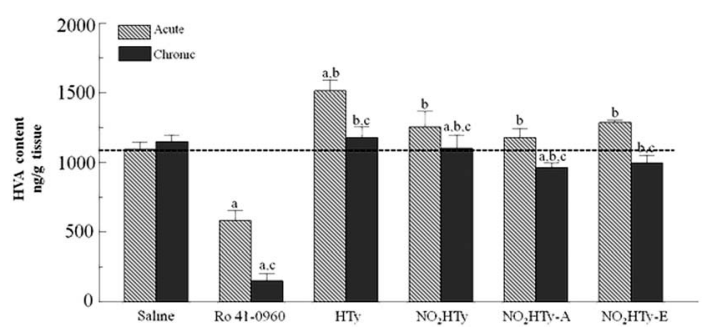

Fig. 4 Effect of the acute and chronic systemic treatments on the striatal content of HVA. Each column represents mean \pm SEM (vertical lines), expressed as $\mathrm{ng} \mathrm{g}^{-1}$ wet tissue $(N=4-8)$. Statistical significance: (a) compared with saline treatment, (b) compared with Ro 41-0960 treatment, and (c) compared with the same treatment under acute conditions. See text for statistical details. decreased DA metabolism and, simultaneously increase DOPAC levels due to monoamine oxidase activity (Fig. 5). We have demonstrated that in HTy and NO-HTy treated animals, there was extensive COMT inhibition in the striatal tissue based on DA and DOPAC levels. In the second generation inhibitors, potency was enhanced with the incorporation of the nitro group. ${ }^{23}$ Although a trend is observed our data does not find a significant difference between the natural precursor phenolic compound (HTy) and the nitroderivative $\left(\mathrm{NO}_{2} \mathrm{HTy}\right)$. HTy may be playing a role in PD as a free radical scavenger, and may not be directly affecting COMT activity. Accordingly, some COMT inhibitors have demonstrated protection against free radical mechanisms that might be involved in PD. In a cell culture model of cerebral ischemia nitecapone and tolcapone are reported to scavenge peroxyl and hydroxyl radicals and inhibit lipid peroxidation ${ }^{32}$ accounting for cytoprotection following hypoxia and hypoglycaemia. ${ }^{33}$ Although $\mathrm{NO}_{2} \mathrm{HTy}$ has already been identified as having antioxidant activity in vitro, ${ }^{26}$ the present paper elucidates the biological effect attributed to nitrocatechol structure on COMT activity of these novel compounds.

Additionally this study shows higher levels of DOPAC from lipophilic nitroderivatives, $\mathrm{NO}_{2} \mathrm{HTy}-\mathrm{A}$ and $\mathrm{NO}_{2} \mathrm{HTy}-\mathrm{E}$, in comparison with their precursor, $\mathrm{NO}_{2} \mathrm{HTy}$. The chemical nature of the side chain substituent is emerging as an important factor in the biological activity of these compounds. Structure-activity relationship studies (SAR) have demonstrated that, although the nitrocatechol structure was mainly responsible for the "anchoring" of the inhibitor to the enzyme active site, variations in the side chain substituent exert a profound influence on both the peripheral selectivity and duration of COMT inhibition. ${ }^{23}$ These results are in accordance with previous studies in which the side chain substituent also exerts an influence on the antioxidant capacity. Regarding nitroester derivatives of HTy, we have demonstrated that antioxidant activity varies depending

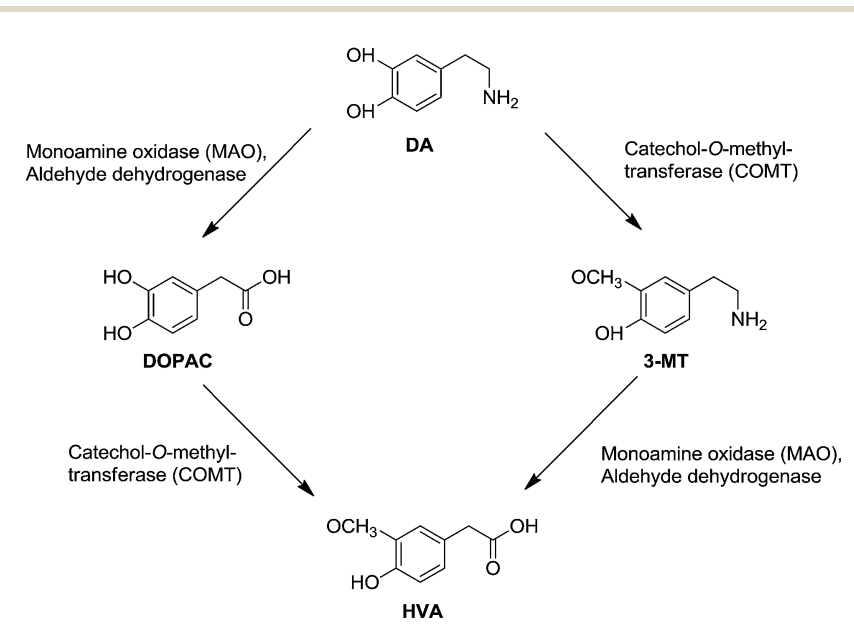

Fig. 5 The metabolism of dopamine. Dopamine can be catabolised by the joint action of the enzymes catechol-O-methyl transferase (COMT), aldehyde dehydrogenase (AH) and monoamine oxidase (MAO). Other abbreviations: dopamine (DA), 3,4-dihydroxyphenylacetic acid (DOPAC), 3-methoxytyramine (3-MT) and homovanillic acid (HVA). 
on the acyl side-chain length; short chains maintained or even enhanced the antioxidant activity compared to nitrohydroxytyrosol. Activity decreased with longer side chains in accordance with the lipophilic nature of these compounds. ${ }^{26}$ This nonlinear association between biological activity and the lipophilic nature of molecules describes the emerging application of lipophilic phenolic compounds in lipid systems. ${ }^{\mathbf{1 1 - 1 3}}$ With this in mind, lipophilic nitroderivatives with short chains ( $\mathrm{NO}_{2} \mathrm{HTy}-\mathrm{A}$ and $\left.\mathrm{NO}_{2} \mathrm{HTy}-\mathrm{E}\right)$ seem to be a suitable option to address biological studies. Moreover, the nitroderivatives' lipohilic nature might also related to the bioavailability and their potential clinical efficacy. At present, entacapone remains the only COMT inhibitor currently used in clinical practice without special restrictions. Nevertheless, it has low oral bioavailability (approximately $35 \%)^{23}$ which explaining the short half-life of this compound. These data seem to be attributed to extensive and variable first-pass metabolism of the current clinical COMT inhibitors. However, phenolic derivatives from olive oil have been previously described as being slowly metabolized by intestinal cells in vitro. Evidence suggests that over $80 \%$ of the precursor HTy that reaches the bloodstream remains a nonmetabolized compound. ${ }^{34}$ Moreover, it has been demonstrated that $\mathrm{NO}_{2} \mathrm{HTy}$ and its lipophilic derivatives are efficiently absorbed in accordance with their lipophilic nature and partially metabolized by intestinal epithelial cell monolayers (unpublished results).

Our results confirmed a significant increase in the striatal content of DA and DOPAC observed for HTy and its derivatives, especially for the nitroderivatives after a chronic treatment in comparison to acute administration. Our results suggest that novel catechols administrated in peripheral tissues can reach the brain after chronic treatment producing longer lasting effects compared with those from acute treatment. Parada and Soares-Da-Silva ${ }^{35}$ demonstrated that the levels of DOPAC and HVA observed in the striatum were not significantly affected by a single dose of nebicapone, but, with higher doses of the inhibitor, an increase in DOPAC and a decrease in HVA levels were observed, indicating that some level of COMT inhibition in the brain may occur at higher doses of the inhibitor; this could explain our results from chronic administration.

For comparison, we carried out experiments with the commercial COMT inhibitor Ro 41-0960. This compound decreased the formation of the striatal HVA with a concomitant increase in striatal DA content as well as DOPAC. This was expected due its proposed activity as an inhibitor of brain COMT activity. This strong inhibition on DA catabolism observed after Ro 41-0960 systemic administration suggests that this compound is a selective and brain penetrant COMT inhibitor. A similar compound, Ro 40-7592 (4'-methyl-3,4dihydroxy-5-nitro-benzophenone) also showed potent and centrally active COMT inhibition. ${ }^{36}$ When comparing acute and chronic treatments, it is important to point out that Ro 41-0960 has similar effects on the striatal content of DA and DOPAC, but HVA striatal content is more affected in the chronic treatment.

DOPAC data showed the higher effect on striatal tissue of Ro 41-0960 inhibitor in comparison to novel compounds. This result suggests that the differences in the potency of the effect may arise from differences in pharmacokinetic behavior. Nitrocatechols have been kinetically characterized as reversible tight-binding inhibitors of COMT. ${ }^{37-39}$ They bind to the enzyme with such a high affinity that the amount of free inhibitor molecules is significantly depleted by the formation of the enzyme-inhibitor complex. ${ }^{\mathbf{4 0}}$

Laatikainen et al. ${ }^{41}$ have studied the gender differences in the effects of COMT inhibition on DA metabolism by tolcapone (30 $\mathrm{mg} \mathrm{kg}^{-1}$, i.p.). In the striatum, they found that tolcapone did not affect DA content, in fact it decreased in female rats. Moreover, they demonstrated a clear increase of DOPAC striatal content in female rats, but not in male rats. In contrast, our data show a clear increase of DA striatal content using male rats (see Fig. 2) and also, a strong DOPAC increase in all treatments (see Fig. 3). In conclusion, with a lower systemically administered dose, a stronger effect was observed with the proposed COMT inhibitors.

In terms of HVA, there was an increase in its intracellular levels, especially in the acute HTy treatment (see Fig. 4). In accordance with this, some authors have described that HTy is converted into homovanillic alcohol (HVAL) by COMT activity ${ }^{\mathbf{2}}$ and this metabolite is subsequently converted into HVA. ${ }^{\mathbf{4 3 , 4 4}} \mathrm{Hu}$ et $a .^{2}{ }^{2}$ offers another explanation that assumes that HTy is part of the DA metabolic pathway, and this is mediated by COMT activity yielding HVAL and HVA as end products. ${ }^{2,44}$ The nitroderivatives of HTy, due to the nitrocatechol structure, do not participate in this metabolism pathway thus there is differential yield in metabolites.

\section{Experimental procedures}

Male albino Wistar rats weighing 270-320 g were used for all the experiments. The rats were kept, three or four rats per cage, at constant room temperature $\left(22 \pm 2{ }^{\circ} \mathrm{C}\right)$, relative humidity $(60 \%)$ with a $12 \mathrm{~h}$ light-dark cycle and food and water ad libitum. Experiments were carried out in accordance with the Guidelines of the European Union Council (2010/63/EU), following the Spanish regulations (BOE 34/11370-421, 2013) for the use of laboratory animals and approved by the Scientific Committee of the University of Seville.

The following drugs were used: 2'-fluoro-3,4-dihydroxy-5nitrobenzophenone (RO 41-0960, Sigma Chemical Co., MO, USA). HTy was extracted and purified from olive oil wastewaters as previously described Fernández-Bolaños et al. ${ }^{45} \mathrm{NO}_{2} \mathrm{HTy}$ and $\mathrm{NO}_{2}$ HTy-A were obtained in our lab accordingly to the synthetic procedures described by Trujillo et al. ${ }^{26} \mathrm{NO}_{2} \mathrm{HTy}$-E was obtained in a similar way from ethyl hydroxytyrosyl ether. ${ }^{10}$

Animals were divided into two main groups: (a) acute treatment: animals received one single dose (20 $\mathrm{mg} \mathrm{kg}^{-1}$; i.p.) between 10:00 and 11:00 a.m. and two hours later they were sacrificed and (b) chronic treatment: animals received a daily dose (20 $\mathrm{mg} \mathrm{kg}^{-1}$; i.p.) for five days between 10:00 and 11:00 a.m. and, the last day, two hours later they were sacrificed. Each main group was divided into six subgroups which were systemically administered saline, Ro 41-0960, HTy, $\mathrm{NO}_{2} \mathrm{HTy}$, $\mathrm{NO}_{2} \mathrm{HTy}-\mathrm{A}$ and $\mathrm{NO}_{2} \mathrm{HTy}-\mathrm{E}$. 
Animals were decapitated between 12:00 and 13:00 $\mathrm{h}$ and the whole brain was quickly removed. The caudoputamen was dissected out, frozen in liquid nitrogen and stored at $-80{ }^{\circ} \mathrm{C}$ until analysis (total time for brain area isolation was $<3 \mathrm{~min}$ ). Postmortem changes of amines were not a problem in this study in light of the relatively short time lapse between decapitation and perchloric acid extraction. The brain tissue was weighed to the nearest milligram and homogenized in $0.1 \mathrm{M}$ perchloric acid ( $1 \mathrm{mg}$ fresh tissue by $30 \mu \mathrm{l}$ perchloric acid) by ultrasonic disintegration over ice using an Hielscher UP100H Ultrasonic Processor (Teltow, Germany). Samples were centrifuged at $30000 \mathrm{~g}$ for $15 \mathrm{~min}$ at $+4{ }^{\circ} \mathrm{C}$ and the supernatant was then filtered through $0.22 \mu \mathrm{m}$ filter nylon (Spin-X centrifuge tube filter, Corning Incorporated, NY, USA). Material was injected by high-pressure injection valve (Rheodyne, CA, USA), with a $10 \mu \mathrm{l}$ sample loop.

Analysis of striatal DA and its metabolites was performed in the isocratic mode by means of HPLC equipped with a VWRHitachi Elite Lachrom L-2130 pump (Tokyo, Japan) in conjunction with a glassy carbon electrode set at $650 \mathrm{mV}$ (DECADE II, Antec Leyden, Netherlands). A Merck Lichrocart cartridge (125 $\mathrm{mm} \times 4 \mathrm{~mm}$ ) column filled with Lichrospher reverse-phase $\mathrm{C}_{18}$ $5 \mu \mathrm{m}$ material was used. The mobile phase consisted of a mixture of $0.05 \mathrm{M}$ of sodium acetate, $0.4 \mathrm{mM}$ of 1-octanesulfonic acid, $0.3 \mathrm{mM}$ of $\mathrm{Na}_{2}$ EDTA and $70 \mathrm{ml}$ methanol per 1 , adjusted to pH 4.1 with acetic acid. The solvent mixtures were filtered by vacuum through a $0.45 \mu \mathrm{m}$ filter, and degassed by vacuum prior to ultrasonic bath in order to eliminate air bubbles, which interfere with the electrochemical detection. All reagents and water were HPLC grade. The flow rate was $1.0 \mathrm{ml} \mathrm{min}^{-1}$.

Concentrations of striatal DA and its metabolites samples were calculated with the aid of eDAQ PowerChrom 280 software.

Results are expressed as mean \pm SEM. Means in the acute and chronic systemic treatments were compared by one-way ANOVA and, where appropriate $(P$ value greater than the 95\% confidence level), followed by the LSD for post hoc multiple range comparisons. An alpha level of 0.05 was used. The Statgraphics Centurion XVI statistical package was used for the analyses.

\section{Conclusion}

The new nitroderivative compounds synthesized from the natural olive oil phenol, hydroxytyrosol, show a remarkable activity in the DA metabolism, suggesting a putative effect against PD as novel and lipophilic COMT inhibitors.

\section{Conflict of interest}

The authors declare no competing financial interest.

\section{Abbreviations}

$\begin{array}{ll}\mathrm{HTy} & \text { Hydroxytyrosol } \\ \mathrm{NO}_{2} \mathrm{HTy} & \text { Nitro-hydroxytyrosol } \\ \mathrm{NO}_{2} \text { HTy-A } & \text { Nitrohydroxytyrosyl acetate }\end{array}$

$\begin{array}{ll}\mathrm{NO}_{2} \mathrm{HTy}-\mathrm{E} & \text { Ethyl nitrohydroxytyrosyl ether } \\ \text { DA } & \text { Dopamine } \\ \text { DOPAC } & \text { 3,4-Dihydroxyphenylacetic acid } \\ \text { HVA } & \text { Homovanillic acid } \\ \text { COMT } & \text { Catechol-O-methyl transferase } \\ \text { i.p } & \text { Intraperitoneal }\end{array}$

\section{Acknowledgements}

This work was supported by the Grant P09-AGR-5098 from Junta de Andalucía. E.G. thanks Junta de Andalucía for a pre-doctoral fellowship. We thank Ms Rianne Stowell for revising the manuscript.

\section{References}

1 F. Visioli and C. Galli, Crit. Rev. Food Sci. Nutr., 2002, 42, 209221.

2 T. Hu, X. W. He, J. G. Jiang and X. L. Xu, J. Agric. Food Chem., 2014, 62, 1449-1455.

3 F. Visioli and E. Bernardini, Curr. Pharm. Des., 2011, 17, 786804.

4 L. Goya, R. Mateos and L. Bravo, Eur. J. Nutr., 2007, 46, 70-78.

5 M. A. Martín, S. Ramos, A. B. Granado-Serrano, M. Rodríguez-Ramiro, M. Trujillo, L. Bravo and L. Goya, Mol. Nutr. Food Res., 2010, 54, 956-966.

6 C. M. Bitler, T. M. Viale, B. Damaj and R. Crea, J. Nutr., 2005, 135, 1475-1479.

7 J. A. González-Correa, M. D. Navas, J. A. Lopez-Villodres, M. Trujillo, J. L. Espartero and J. P. De La Cruz, Neurosci. Lett., 2008, 446, 143-146.

8 R. Bernini, F. Crisante, M. Barontini, D. Tofani, V. Balducci and A. Gambacorta, J. Agric. Food Chem., 2012, 60, 740-816.

9 M. Trujillo, R. Mateos, L. Collantes De Teran, J. L. Espartero, R. Cert, M. Jover, F. Alcudia, J. Bautista, A. Cert and J. Parrado, J. Agric. Food Chem., 2006, 54, 3779-3785.

10 A. Madrona, G. Pereira-Caro, R. Mateos, G. Rodríguez, M. Trujillo, J. Fernández-Bolaños and J. L. Espartero, Molecules, 2009, 14, 1762-1772.

11 L. Liu, C. Jin and Y. Zhang, RSC Adv., 2014, 4, 2879-2891.

12 M. Laguerre, J. Lecomte and P. Villeneuve, Lipid Technol., 2014, 26, 59-62.

13 M. Laguerre, C. Bayrasy, A. Panya, J. Weiss, D. J. McClements, J. Lecomte, E. A. Decker and P. Villeneuve, Crit. Rev. Food Sci. Nutr., 2015, 55, 183-201.

14 R. Winkler and C. Hertweck, ChemBioChem, 2007, 8, 973977.

15 K. S. Ju and R. E. Parales, Microbiol. Mol. Biol. Rev., 2010, 74, 250-272.

16 K. B. Gavazov, Acta Chim. Slov., 2012, 59, 1-17.

17 J. Borgulya, M. Da Prada, J. Dingemanse, R. Scherschlicht, B. Schlappi and G. Zurcher, Drugs Future, 1991, 16, 719-721.

18 A. Gordin, S. Kaakkola and H. Teravainen, J. Neural Transm., 2004, 111, 1343-1363.

19 F. Assal, L. Spahr, A. Hadengue, L. Rubbici-Brandt and P. R. Burkhard, Lancet, 1998, 352, 958. 
20 C. Colissimo, J. Neurol., 1999, 246, 880-882.

21 S. A. Parashos, C. L. Wielinski and J. A. Kern, Clin. Neuropharmacol., 2004, 27, 119-123.

22 L. Almeida and P. Soares-da-Silva, Drugs R\&D, 2003, 4, 207217.

23 M. J. Bonifácio, P. N. Palma, L. Almeida and P. Soares-daSilva, CNS Drug Rev., 2007, 13, 352-379.

24 L. E. Kiss, H. S. Ferreira, L. Torrão, M. J. Bonifácio, P. N. Palma, P. Soares-da-Silva and D. A. Learmonth, $J$. Med. Chem., 2010, 53, 3396-3411.

25 O. Kambur, P. T. Männistö, A. M. Pusa, M. Käenmäki, E. A. Kalso and V. K. Kontinen, Eur. J. Pain, 2011, 15, 732740 .

26 M. Trujillo, E. Gallardo, A. Madrona, L. Bravo, B. Sarriá, J. A. González-Correa, R. Mateos and J. L. Espartero, J. Agric. Food Chem., 2014, 62, 10297-10303.

27 B. Sarriá, R. Mateos, E. Gallardo, S. Ramos, M. A. Martín, L. Bravo and L. Goya, Food Chem. Toxicol., 2012, 50, 37523758.

28 T. Keranen, A. Gordin, V. P. Harjola, M. Karlsson, K. Korpela, P. J. Pentikainen, H. Rita, L. Seppala and T. Wikberg, Eur. J. Clin. Pharmacol., 1994, 46, 151-157.

29 J. Dingemanse, K. Jorga, M. Schmitt, R. Gieschke, B. Fotteler, G. Zurcher, M. Da Prada and P. van Brummelen, Clin. Pharmacol. Ther., 1995, 57, 508-517.

30 D. A. Learmonth, M. A. Vieira-Coelho, J. Benes, P. C. Alves, N. Borges, A. P. Freitas and P. Soares-da-Silva, J. Med. Chem., 2002, 45, 685-695.

31 D. A. Learmonth, P. Nuno Palma, M. A. Vieira-Coelho and P. Soares-da-Silva, J. Med. Chem., 2004, 47, 6207-6217.
32 Y. J. Suzuki, M. Tsuchiya, A. Safadi, V. E. Kagan and L. Packer, Free Radical Biol. Med., 1992, 13, 517-525.

33 J. Ekblom, H. Garpenstrand, O. Tottmar, J. A. Prince and L. Oreland, J. Neural Transm., 1998, 52, 93-98.

34 R. Mateos, G. Pereira-Caro, S. Saha, R. Cert, M. RedondoHorcajo, L. Bravo and P. A. Kroon, Food Chem., 2011, 125, 865-872.

35 A. Parada and P. Soares-Da-Silva, Pharmacology, 2003, 68, 29-37.

36 S. Kaakkola and R. J. Wurtman, Brain Res., 1992, 587, 241249.

37 N. Borges, M. A. Vieira-Coelho, A. Parada and P. Soares-daSilva, J. Pharmacol. Exp. Ther., 1997, 282, 812-817.

38 T. Lotta, J. Vidgren, C. Tilgmann, I. Ulmanen, K. Melen, I. Julkunen and J. Taskinen, Biochemistry, 1995, 34, 42024210.

39 E. Schultz and E. Nissinen, Biochem. Pharmacol., 1989, 38, 3953-3956.

40 R. A. Copeland, Enzymes: A practical introduction to structure, mechanism and data analysis, John Wiley \& Sons, Inc., New York, 2nd edn, 2000, pp. 305-317.

41 L. M. Laatikainen, T. Sharp, P. J. Harrison and E. M. Tunbridge, PLoS One, 2013, 8, e61839.

42 E. Gallardo, R. Palma-Valdés, J. L. Espartero and M. Santiago, Neurosci. Lett., 2014, 579, 173-176.

43 D. Caruso, F. Visioli, R. Patelli, C. Galli and G. Galli, Metabolism, 2001, 50, 1426-1428.

44 R. De la Torre, Inflammopharmacology, 2008, 16, 245-247.

45 J. Fernandez-Bolaños, A. Heredia, G. Rodriguez, R. Rodriguez, A. Jimenez and R. Guillen, US Pat., 6849,770 B2, 2005. 